\title{
Correlation Transfer and Diffusion of Ultrasound-Modulated Multiply Scattered Light
}

\author{
Sava Sakadžić and Lihong V. Wang* \\ Optical Imaging Laboratory, Department of Biomedical Engineering, Texas A\&M University, \\ 3120 TAMU, College Station, Texas 77843-3120, USA
}

(Received 4 January 2006; published 26 April 2006)

\begin{abstract}
We develop a temporal correlation transfer equation (CTE) and a temporal correlation diffusion equation (CDE) for ultrasound-modulated multiply scattered light. These equations can be applied to an optically scattering medium with embedded optically scattering and absorbing objects to calculate the power spectrum of light modulated by a nonuniform ultrasound field. We present an analytical solution based on the CDE and Monte Carlo simulation results for light modulated by a cylinder of ultrasound in an optically scattering slab. We further validate with experimental measurements the numerical calculations for an actual ultrasound field. The CTE and CDE are valid for moderate ultrasound pressures and on a length scale comparable with the optical transport mean-free path. These equations should be applicable to a wide spectrum of conditions for ultrasound-modulated optical tomography of soft biological tissues.
\end{abstract}

PACS numbers: 42.25.Dd, 42.90.+m

Considerable effort has been expended recently to advance soft biological tissue imaging based on ultrasoundmodulated multiply scattered light. The optical properties of tissues in visible and near-infrared regions are related to their molecular structure. Radiation at these wavelengths is nonionizing with the potential for the functional imaging and detection of tissue abnormalities. However, due to the diffusion of light, it is difficult to achieve simultaneously both good resolution and good imaging depth in pure optical imaging modalities such as optical coherence tomography or diffuse optical tomography [1].

Ultrasound-modulated optical tomography (UOT) is a hybrid technique which combines ultrasonic resolution and optical contrast. It is being proposed in order to provide better resolution in the optical imaging of soft biological tissues at imaging depths where light is completely diffused. With this technique [2], optical radiation, which has high temporal coherence, and focused ultrasound are applied simultaneously to the imaging target. The intensity of the ultrasound-modulated light is related to the optical properties of the tissue in the interaction region of the ultrasonic and electromagnetic waves.

Efficient detection of ultrasound-modulated optical intensity is challenging because of diffused light propagation and uncorrelated phases among optical speckles. At present, the development of effective detection systems is the subject of intense research [3,4]. Simultaneously, a theoretical understanding of the ultrasound modulation of multiply scattered light is emerging. Similar to dynamic light scattering by scatterers undergoing Brownian motion [5], dynamic scattering by optical scatterers oscillating in an ultrasound field causes optical frequency shifts [6]. In addition, shifts are produced by the ultrasound-induced changes of the optical index of refraction $[7,8]$. The theoretical model [8], which combines both mechanisms of modulation, has been subsequently extended to account for anisotropic optical scattering [9] and Brownian motion $[9,10]$. In addition, Ref. [11] accounts for strong correla- tions between ultrasound-induced optical phase increments which exist when the ratio of optical transport mean-free path $l_{\text {tr }}$ to ultrasound wavelength $\lambda_{a}$ is small. An existing Monte Carlo algorithm [12] can be adapted to explore complex geometries.

Existing theoretical models are based on the diffusingwave spectroscopy (DWS) approach [5,13], where the interaction of a plane ultrasound wave with diffused light is considered in an infinite scattering medium. As a result, applications are limited to simple geometries where the ultrasound field can be approximated as a plane wave and where the probability density function of the optical path length is analytically known. Consequently, only transmission through $[8,9,11]$, and reflection from $[10,11]$, an infinite scattering slab filled with ultrasound have been analytically studied. In practice, however, both the ultrasound field and the optical parameters are heterogeneous, and a more general theoretical model is needed.

In this Letter, we formally derive a temporal correlation transfer equation (CTE) and a temporal correlation diffusion equation (CDE) for the ultrasound-modulated multiply scattered light for isotropic optical scattering and $k_{a} l_{\text {tr }} \gg 1$, where $k_{a}=2 \pi / \lambda_{a}$. These equations can be used to obtain both analytical and numerical solutions for the distribution of the modulated light intensity in scattering samples with heterogeneous optical parameters and a nonuniform ultrasound field. In addition, simple forms of CTE and CDE benefit from all of the mathematical tools available for the radiative transfer and diffusion equations. A derivation of a more complex CTE based on the ladder approximation of the Bethe-Salpeter equation will be presented elsewhere [14].

We first confirm the agreement between the analytical solution for the scattering slab filled with ultrasound based on the previous DWS approach and the simple solution of CDE. We further provide both analytical and Monte Carlo solutions for the more practical configuration where a cylinder of ultrasound insonifies a scattering slab. Finally, 
the experimental results for a similar configuration are compared with the calculation based on the finitedifference model of CDE.

We consider the interaction of ultrasound with monochromatic light that diffuses through the medium with discrete, uncorrelated optical scatterers. We further assume independent scattering and neglect the polarization for simplicity. Under the weak scattering approximation that the optical mean-free path is much greater than the optical wavelength, transfer of light can be described by ladder diagrams [15]. In our case, this also involves calculation of the optical phase increments due to both mechanisms of ultrasound modulation along the optical paths. The phase increments are generally correlated if they originate at positions separated by less than $l_{\text {tr }}$ or $\lambda_{a}$ [11], which creates difficulties in the derivation of a simple transferlike equation for the temporal correlation of ultrasound-modulated light [14].

However, a simple form of CTE can be obtained when $k_{a} l_{\text {tr }} \gg 1$. At scales larger than $l_{\text {tr }}$, the effect of ultrasound modulation can be calculated by assuming isotropic scattering, where $l_{\text {tr }}$ is used instead of the mean-free path $[9,11,12]$. The condition $k_{a} l_{\text {tr }} \gg 1$ then ensures that the ultrasound-induced optical phase increments associated with the different scattering events are independent. The only correlation between phase increments which then exists is between (i) the phase increment due to the index of refraction changes along the free path and (ii) the phase increments due to displacements of these two scatterers along the free path [11]. This allows for a simple form of the CTE that is valid on the scale comparable with $l_{\mathrm{tr}}$. In soft biological tissues, $l_{\text {tr }} \approx 1 \mathrm{~mm}$ for visible and nearinfrared light and $k_{a} l_{\text {tr }}>10$ for ultrasound frequencies greater than $2.4 \mathrm{MHz}$.

Consider optical scatterers at resting positions $\mathbf{r}_{a}$ and $\mathbf{r}_{b}$ and assume that the ultrasound field in volumes of $\sim l_{\text {tr }}^{3}$ can be locally approximated as a plane wave $P(\mathbf{r}, t)=$ $P_{0} \cos \left(\omega_{a} t-\mathbf{k}_{a} \cdot \mathbf{r}+\phi\right)$, where $\mathbf{k}_{a}=k_{a} \hat{\mathbf{\Omega}}_{a}$, and $P_{0}$, $\omega_{a}, \hat{\boldsymbol{\Omega}}_{a}$, and $\phi$ are the pressure amplitude, the angular frequency, the propagation direction of the ultrasound $\left(\left|\hat{\boldsymbol{\Omega}}_{a}\right|=1\right)$, and the local initial phase, respectively. For moderate ultrasound pressures, the optical index of refraction experiences a small perturbation approximated with $n(\mathbf{r}, t)=n_{0}\left[1+\eta P(\mathbf{r}, t) /\left(\rho v_{a}^{2}\right)\right]$, where $\rho$ is the fluid density, $v_{a}$ is the ultrasound speed, and $\eta$ is the elasto-optical coefficient. We obtain the increment $\delta=k_{0} n_{0}\left|\mathbf{r}_{b}-\mathbf{r}_{a}\right|+$ $\varphi_{a, b}(t)$ of the optical phase along the free path between $\mathbf{r}_{a}$ and $\mathbf{r}_{b}$ by integrating the $k_{0} n(\mathbf{r}, t)$ along the path, where

$$
\varphi_{a, b}(t)=k_{0} n_{0}\left[\hat{\mathbf{\Omega}} \cdot\left[\mathbf{e}_{b}(t)-\mathbf{e}_{a}(t)\right]+\frac{\eta}{\rho v_{a}^{2}} \int_{\mathbf{r}_{a}}^{\mathbf{r}_{b}} P(\mathbf{r}, t) d r\right],
$$

and $k_{0}$ is the optical wave number in vacuum. In Eq. (1), we approximate the distance between scatterers with $\left|\mathbf{r}_{b}-\mathbf{r}_{a}\right|+\hat{\boldsymbol{\Omega}} \cdot\left[\mathbf{e}_{b}(t)-\mathbf{e}_{a}(t)\right]$, where $\hat{\boldsymbol{\Omega}}\left|\mathbf{r}_{b}-\mathbf{r}_{a}\right|=\mathbf{r}_{b}-\mathbf{r}_{a}$, $\mathbf{e}_{s}(t)=\hat{\boldsymbol{\Omega}}_{a} P_{0} S_{a} /\left(k_{a} \rho v_{a}^{2}\right) \sin \left(\omega_{a} t-\mathbf{k}_{a} \cdot \mathbf{r}_{s}+\phi-\phi_{a}\right)$ is the ultrasound-induced displacement of the optical scatterer at $\mathbf{r}_{s}(s=a, b)$, and $S_{a}$ and $\phi_{a}$ are, respectively, deviations of the amplitude and phase of the scatterer from the motion of the surrounding fluid [11]. The second term in Eq. (1) is the phase increment due to the ultrasound-induced index of refraction changes. The scatterer displacement in the integration limits is neglected since $\left|\mathbf{e}_{s}(t)\right| \ll l_{\text {tr }}$ at the relatively high ultrasound frequencies and moderate pressures which are assumed in this calculation. However, at ultrasound frequencies greater than several tens of megahertz when $P_{0}>10^{5} \mathrm{~Pa}$, integration along straight lines might be inappropriate due to optical wavefront distortion [11].

We assume that the electrical field mutual coherence function $\left\langle E\left(\mathbf{r}_{b^{\prime}}, t\right) E^{*}\left(\mathbf{r}_{b^{\prime \prime}}, t+\tau\right)\right\rangle$ is quasiuniform, and we relate it to the time-varying specific intensity $I\left(\mathbf{r}_{b}, \hat{\mathbf{\Omega}}, t, \tau\right)$ by a spatial Fourier transform over the difference variable $\mathbf{r}_{b^{\prime}}-\mathbf{r}_{b^{\prime \prime}}$ in the center-of-gravity coordinate system [1618], where $\mathbf{r}_{b}=\left(\mathbf{r}_{b^{\prime}}+\mathbf{r}_{b^{\prime \prime}}\right) / 2$, and \langle\rangle denotes the ensemble averaging. Let $E\left(\mathbf{r}_{a}, t\right)$ be the partial wave scattered at $\mathbf{r}_{a}$ toward $\mathbf{r}_{b}$. For isotropic scattering and $k_{a} l_{\text {tr }} \gg 1$, there is no correlation between $\varphi_{a, b}(t)$ and the other ultrasound-induced optical phase increments accumulated in $E\left(\mathbf{r}_{a}, t\right)$, and $I\left(\mathbf{r}_{b}, \hat{\mathbf{\Omega}}, t, \tau\right)$ is independent of time $t$. The phase term $\Delta \varphi=\varphi_{a, b}(t+\tau)-\varphi_{a, b}(t)$ satisfies $\Delta \varphi \ll 1$ for $l_{\mathrm{tr}} \approx 1 \mathrm{~mm}$ and $P_{0}<10^{5} \mathrm{~Pa}$, and we approximate $\exp (i \Delta \varphi)$ with $1-\left|\mathbf{r}_{b}-\mathbf{r}_{a}\right| l_{\mathrm{tr}}^{-1}\left\langle\Delta \varphi^{2}\right\rangle_{t_{\mathrm{tr}}} / 2$. For isotropic scattering, $l_{\mathrm{tr}}^{-1}=\mu_{t}$ and $\mu_{t}=\mu_{s}+\mu_{a}$, where $\mu_{t}, \mu_{s}$, and $\mu_{a}$ are the optical extinction, scattering, and absorption coefficients, respectively. $\left\langle\Delta \varphi^{2}\right\rangle_{l_{\text {tr }}}$ is the average value of $\Delta \varphi^{2}$ in volume $\sim l_{\text {tr }}^{3}$ per mean optical free path. From the probability density of the free path $l$, which is $l_{\text {tr }}^{-1} \exp \left(-l / l_{\text {tr }}\right)$, we obtain

$$
\begin{aligned}
\left\langle\Delta \varphi^{2}\right\rangle_{l_{\mathrm{tr}}}= & \Lambda^{2} \sin ^{2}\left(\frac{1}{2} \omega_{a} \tau\right) \frac{\left(l_{\mathrm{tr}} \mathbf{k}_{a} \cdot \hat{\mathbf{\Omega}}\right)^{2}}{1+\left(l_{\mathrm{tr}} \mathbf{k}_{a} \cdot \hat{\mathbf{\Omega}}\right)^{2}} \\
& \times\left[S_{a}^{2}\left(\hat{\mathbf{\Omega}} \cdot \hat{\mathbf{\Omega}}_{a}\right)^{2}+\eta^{2} /\left(\hat{\mathbf{\Omega}} \cdot \hat{\mathbf{\Omega}}_{a}\right)^{2}-2 \eta S_{a} \cos \left(\phi_{a}\right)\right],
\end{aligned}
$$

where $\Lambda=2 k_{0} n_{0} P_{0} /\left(k_{a} \rho v_{a}^{2}\right)$. The three terms in square brackets in Eq. (2) are related to the two mechanisms of modulation and the correlation between the phase increments produced by these mechanisms along the same free path, respectively [11]. The increment of the intensity $I\left(\mathbf{r}_{b}, \hat{\mathbf{\Omega}}, \tau\right)$ that is due to the contribution of $I\left(\mathbf{r}_{a}, \hat{\mathbf{\Omega}}^{\prime}, \tau\right)$, which is scattered at $\mathbf{r}_{a}$ into direction $\hat{\mathbf{\Omega}}$, is equal to $\Delta I=$ $I\left(\mathbf{r}_{a}, \hat{\mathbf{\Omega}}^{\prime}, \tau\right) \exp \left(-\mu_{t}\left|\mathbf{r}_{b}-\mathbf{r}_{a}\right|\right)\left[1-\left|\mathbf{r}_{b}-\mathbf{r}_{a}\right| \mu_{t}\left\langle\Delta \varphi^{2}\right\rangle_{l_{\mathrm{tr}}} / 2\right]$. By accumulating all of the increments along the $\hat{\boldsymbol{\Omega}}$ direction starting from some distant $\mathbf{r}_{0}$, we have

$$
\begin{aligned}
I\left(\mathbf{r}_{b}, \hat{\boldsymbol{\Omega}}, \tau\right)= & I_{0}\left(\mathbf{r}_{b}, \hat{\boldsymbol{\Omega}}, \tau\right) \\
& +\int_{\mathbf{r}_{0}}^{\mathbf{r}_{b}} \int_{4 \pi} \mu_{s} p\left(\hat{\boldsymbol{\Omega}}, \hat{\boldsymbol{\Omega}}^{\prime}\right) \Delta I d\left|\mathbf{r}_{b}-\mathbf{r}_{a}\right| d \hat{\mathbf{\Omega}}^{\prime},
\end{aligned}
$$

where $I_{0}\left(\mathbf{r}_{b}, \hat{\mathbf{\Omega}}, \tau\right)$ is due to the unscattered field [17]. After applying $\hat{\boldsymbol{\Omega}} \cdot \nabla$ to Eq. (3), we obtain the CTE as 


$$
\begin{aligned}
\hat{\mathbf{\Omega}} \cdot \nabla I(\mathbf{r}, \hat{\mathbf{\Omega}}, \tau)= & -\left(\mu_{a}+\mu_{s}\right) I(\mathbf{r}, \hat{\mathbf{\Omega}}, \tau)+S(\mathbf{r}, \hat{\mathbf{\Omega}}) \\
& +\mu_{s} \int_{4 \pi} p\left(\hat{\mathbf{\Omega}}, \hat{\mathbf{\Omega}}^{\prime}\right)\left[1-\frac{1}{2}\left\langle\Delta \varphi^{2}\right\rangle_{l_{\mathrm{tr}}}\right] \\
& \times I\left(\mathbf{r}, \hat{\mathbf{\Omega}}^{\prime}, \tau\right) d \mathbf{\Omega}^{\prime} .
\end{aligned}
$$

In Eq. (4), $p\left(\hat{\mathbf{\Omega}}, \hat{\mathbf{\Omega}}^{\prime}\right)=1 /(4 \pi)$ is the isotropic scattering phase function, and $S(\mathbf{r}, \hat{\boldsymbol{\Omega}})$ is the monochromatic source term. Like in the case of Brownian motion [18], this equation can be obtained by preaveraging the phase increments in a more rigorously derived CTE [14].

To obtain the CDE, we apply the standard approximation $I(\mathbf{r}, \hat{\mathbf{\Omega}}, \tau) \approx[\Phi(\mathbf{r}, \tau)+3 \hat{\mathbf{\Omega}} \cdot \mathbf{J}(\mathbf{r}, \tau)] /(4 \pi)$ in Eq. (4). $\Phi(\mathbf{r}, \tau)$ is actually the temporal field autocorrelation function related to the optical power spectrum by the temporal Fourier transform. The CDE is

$$
\nabla \cdot[D \nabla \Phi(\mathbf{r}, \tau)]-\left[\mu_{a}+\mu_{s} \hat{\varphi}(\tau)\right] \Phi(\mathbf{r}, \tau)=-S_{0}(\mathbf{r}) .
$$

In Eq. (5), $D=\left(3 \mu_{s}\right)^{-1}$, and $\hat{\varphi}(\tau)$ is given by

$$
\begin{aligned}
\hat{\varphi}(\tau)= & \left(\Lambda^{2} / 2\right) \sin ^{2}\left(\omega_{a} \tau / 2\right)\left[\eta^{2}\left(k_{a} l_{\mathrm{tr}}\right) \tan ^{-1}\left(k_{a} l_{\mathrm{tr}}\right)\right. \\
& \left.+S_{a}^{2} / 3-2 \eta S_{a} \cos \left(\phi_{a}\right)\right] .
\end{aligned}
$$

Brownian motion could also be considered by including $2 D_{B} k_{0}^{2} \mu_{s} \tau$ in addition to $\mu_{s} \hat{\varphi}(\tau)$ in Eq. (5), where $D_{B}$ is an appropriate diffusion constant [19].

In infinite media, the solution of Eq. (5) for monochromatic point source $S_{0}$ at the origin is

$$
\Phi(\mathbf{r}, \tau)=S_{0}(4 \pi D)^{-1} \exp \left(-r \sqrt{\left[\mu_{a}+\mu_{s} \hat{\varphi}(\tau)\right] / D}\right) / r,
$$

and it can be used to study analytically various configurations of ultrasound within the scattering media. In finite media, the boundary conditions are identical to those in the diffusion equation used in radiative transfer [20]. For matched optical properties, continuity requires that $\Phi(\mathbf{r}, \tau)$ and $D \partial \Phi(\mathbf{r}, \tau) / \partial n$ are constant across the boundary, where $\hat{\mathbf{n}}$ is a unit vector perpendicular to the boundary. For a scattering half-space $(z>0)$ filled with ultrasound and irradiated by a pencil source from the free space, the boundary condition is $\Phi(\mathbf{r}, \tau)=2 D \partial \Phi(\mathbf{r}, \tau) / \partial z$, which leads to the extrapolated zero boundary position at $z=$ $-2 D$.

Equation (5) can be solved analogously to the diffusion equation of fluence rate [21] for $\Phi(\tau)$, which is $\Phi(\mathbf{r}, \tau)$ integrated over the whole transmission surface of the scattering slab of thickness $L$ filled with ultrasound and irradiated with the pencil monochromatic source $S_{0}$

$$
\Phi(\tau)=\frac{3 S_{0}}{4 \pi} \frac{\sinh \left[\left(z_{0}+2 D\right) \sqrt{\left(\mu_{a}+\mu_{s} \hat{\varphi}(\tau)\right) / D}\right]}{\sinh \left[(L+4 D) \sqrt{\left(\mu_{a}+\mu_{s} \hat{\varphi}(\tau)\right) / D}\right]} .
$$

In Eq. (8), $z_{0}=1 / \mu_{s}$ is the depth of the converted isotropic source and the solution is, up to the normalization constant, identical to the one obtained earlier $[8,9,11]$.

Next we consider an infinitely wide scattering slab, with surface planes at $x=0 \mathrm{~mm}$ and $x=20 \mathrm{~mm}$. We assume $v_{a}=1480 \mathrm{~m} / \mathrm{s}, \quad \rho=10^{3} \mathrm{~kg} / \mathrm{m}^{3}, \quad \eta=0.32, \quad \mu_{a}=$ $0.1 \mathrm{~cm}^{-1}$, isotropic scattering with $\mu_{s}=10 \mathrm{~cm}^{-1}, n_{0}=$ 1.33 in whole space, $S_{a}=1$, and $\phi_{a}=0$, as typical values for soft biological tissues and visible and near-infrared light [11]. A cylinder of radius $a=3.175 \mathrm{~mm}$, infinitely long in the $Z$ direction, with an axis at $(x, y)=$ $(10 \mathrm{~mm}, 0 \mathrm{~mm})$ is filled with a $5 \mathrm{MHz}$ ultrasound of pressure amplitude $P_{0}=10^{5} \mathrm{~Pa}$ traveling in the $Z$ direction. A pencil light source $S_{0}$ of wavelength $\lambda_{0}=532 \mathrm{~nm}$ irradiates the slab along the $X$ direction at $(x, y, z)=$ $(0 \mathrm{~mm}, 10 \mathrm{~mm}, 0 \mathrm{~mm})$. Equation (5) has solutions $\Phi_{\text {inc }}\left(\mathbf{r}_{d}, \tau\right), \Phi_{\mathrm{sc}}\left(\mathbf{r}_{d}, \tau\right)$, and $\Phi_{\mathrm{in}}\left(\mathbf{r}_{d}, \tau\right)$, which are for the autocorrelation functions incident from the source, scattered from the cylinder, and inside the cylinder, respectively [22]. If the cylinder axis is at the origin, then

$$
\Phi_{\{\ldots\}}\left(\mathbf{r}_{d}, \tau\right)=\sum_{n=0}^{+\infty} \cos \left(n \phi_{d}\right) \int_{0}^{+\infty} \cos \left(p z_{d}\right) \Psi_{\{\ldots\}}(p) d p,
$$

where $\Psi_{\text {inc }}(p)=H_{n}\left(x_{>}\right) I_{n}\left(x_{<}\right), \quad \Psi_{\mathrm{sc}}(p)=B_{n}(p) K_{n}(x)$, and $\Psi_{\text {in }}(p)=C_{n}(p) I_{n}(y) ; \quad \mathbf{r}_{s}=\left(\rho_{s}, \phi_{s}, z_{s}\right)$ and $\mathbf{r}_{d}=$ $\left(\rho_{d}, \phi_{d}, z_{d}\right)$ are positions of the point source and the detector in the cylindrical coordinates; $I_{n}$ and $K_{n}$ are modified Bessel functions of the first and second kind, respectively; $\quad x_{\lessgtr}=\rho_{\lessgtr} \sqrt{p^{2}-k_{\text {out }}^{2}} ; \quad \rho_{\lessgtr}=\min (\max ) \times$ $\left[\rho_{s}, \rho_{d}\right] ; \quad x=\rho_{d} \sqrt{p^{2}-k_{\mathrm{out}}^{2}} ; \quad y=\rho_{d} \sqrt{p^{2}-k_{\mathrm{in}}^{2}} ; \quad k_{\mathrm{in}}^{2}=$ $-\left[\mu_{a}+\mu_{s} \hat{\varphi}(\tau)\right] / D ; k_{\text {out }}^{2}=-\mu_{a} / D ; B_{n}$ and $C_{n}$ are given by

$$
\begin{aligned}
& B_{n}(p)=-H_{n}\left(z_{b}\right) \frac{x_{b} I_{n}^{\prime}\left(x_{b}\right) I_{n}\left(y_{b}\right)-y_{b} I_{n}^{\prime}\left(y_{b}\right) I_{n}\left(x_{b}\right)}{x_{b} K_{n}^{\prime}\left(x_{b}\right) I_{n}\left(y_{b}\right)-y_{b} I_{n}^{\prime}\left(y_{b}\right) K_{n}\left(x_{b}\right)}, \\
& C_{n}(p)=-H_{n}\left(z_{b}\right) \frac{x_{b} I_{n}^{\prime}\left(x_{b}\right) K_{n}\left(x_{b}\right)-x_{b} K_{n}^{\prime}\left(x_{b}\right) I_{n}\left(x_{b}\right)}{x_{b} K_{n}^{\prime}\left(x_{b}\right) I_{n}\left(y_{b}\right)-y_{b} I_{n}^{\prime}\left(y_{b}\right) K_{n}\left(x_{b}\right)},
\end{aligned}
$$

where $H_{n}\left(z_{b}\right)=\left[(\operatorname{sgn}(n)+1) S_{0} K_{n}\left(z_{b}\right)\right] /\left(2 \pi^{2} D\right), \quad x_{b}=$ $a \sqrt{p^{2}-k_{\mathrm{out}}^{2}}, y_{b}=a \sqrt{p^{2}-k_{\mathrm{in}}^{2}}, z_{b}=\rho_{s} \sqrt{p^{2}-k_{\mathrm{out}}^{2}}$, and $\operatorname{sgn}(n)$ is the sign function. We use Eq. (9) to obtain values for the modulation depth (MD), defined as the amplitude ratio of the first to the zeroth harmonics of the modulated light [Fig. 1(a)]. Three pairs of independent cylinder images [21] are used to satisfy the boundary conditions. The analytical solution agrees with the Monte Carlo solution (MCS) [9,12], modified for the cylindrical object [Fig. 1(b)]. In Fig. 1(c), the two solutions are compared along the $Y$ direction on the slab surfaces. The MD is higher away from the source, in the shadow of the cylinder, due to the counteracting contributions of the modulated and unmodulated light.

In the experiment, we immersed in water a wide, $20 \mathrm{~mm}$ thick slab, with $\mu_{a}=0.1 \mathrm{~cm}^{-1}$ and reduced scattering coefficient $\mu_{s}=10 \mathrm{~cm}^{-1}$, made of agar, Lyposine $20 \%$, and Trypan Blue dye. A flat ultrasound transducer with a $5-\mathrm{MHz}$ frequency and a $3.175-\mathrm{mm}$ radius was positioned, as in the theoretical model, with a surface at $z=-50 \mathrm{~mm}$. A $10^{5} \mathrm{~Pa}$ pressure amplitude was measured at $z=0 \mathrm{~mm}$ with a needle hydrophone on the acoustic axis. We used a 

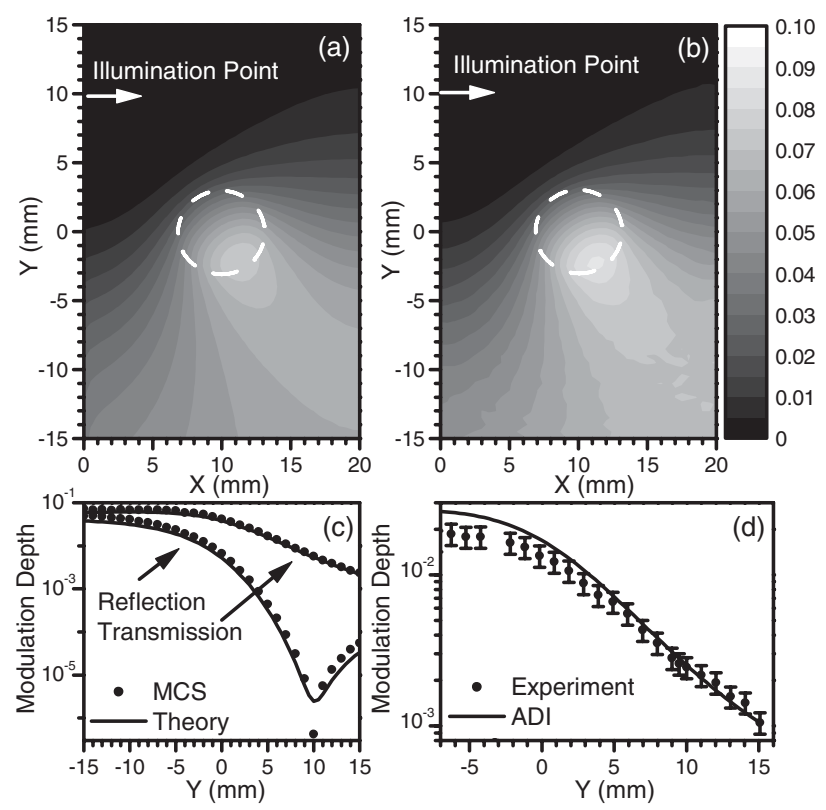

FIG. 1. MD of the ultrasound-modulated light for an ultrasound cylinder in a scattering slab. (a),(b) MD obtained analytically and with MCS, respectively, at $z=0 \mathrm{~mm}$. The white circles mark the ultrasound cross section. (c) MD obtained analytically and with MCS for $z=0 \mathrm{~mm}$ at the transmission $(x=20 \mathrm{~mm})$ and reflection $(x=0 \mathrm{~mm})$ planes. (d) MD measured experimentally and calculated using the ADI for $z=$ $0 \mathrm{~mm}$ at the transmission plane.

previously described setup [4] to measure the modulation depth at the transmission plane of the slab. The measured data [Fig. 1(d)] were in agreement with the numerical calculation, which used an alternating direction implicit algorithm (ADI) adapted for Eq. (5). For the ADI, the nonuniform ultrasound field was calculated using the program FIELDII [23], where transducer apodization and amplitude were adjusted to match the parameters of the real transducer measured by the hydrophone. The cell size was set to $1 / 3 \mathrm{~mm}$ in order to model appropriately the boundary conditions.

In conclusion, we derived the CTE and the CDE for ultrasound-modulated light which is valid for optical and ultrasound spatial inhomogeneities on the order of $l_{\text {tr }}$, for moderate ultrasound pressures and frequencies satisfying $k_{a} l_{\text {tr }} \gg 1$. CDE could be of use for the estimation of sensitivity and signal-to-noise ratios in UOT, where both heterogeneous ultrasound fields and optical parameters are encountered. It can be solved analytically or numerically by the many methods developed for the diffusion equation. This permitted us to obtain agreement, for the first time, between the theoretical model and the experimental measurement of the modulation depth of ultrasound-modulated light in strongly scattering media. More challenging setups with highly focused ultrasound and with very high ultrasound pressure should be the subject of further theoretical development.

Thanks to A. Garcia-Uribe for measuring the optical properties and to R. Zemp for helpful discussion.
This project was sponsored by NIH Grant No. R33 CA 094267.

*Electronic address: LWang@tamu.edu

[1] B.E. Bouma and G. J. Tearney, Handbook of Optical Coherence Tomography (Marcel Dekker, New York, 2001); A. Gibson, J. Hebden, and S. Arridge, Phys. Med. Biol. 50, R1 (2005).

[2] F. A. Marks, H. W. Tomlinson, and G. W. Brooksby, Proc. SPIE-Int. Soc. Opt. Eng. 1888, 500 (1993); L. Wang, S. L. Jacques, and X. Zhao, Opt. Lett. 20, 629 (1995).

[3] L. V. Wang and G. Ku, Opt. Lett. 23, 975 (1998); S. Leveque, A. C. Boccara, M. Lebec, and H. Saint-Jalmes, Opt. Lett. 24, 181 (1999); G. Yao, S. Jiao, and L. V. Wang, Opt. Lett. 25, 734 (2000); A. Lev, Z. Kotler, and B. G. Sfez, Opt. Lett. 25, 378 (2000); J. Li, G. Ku, and L. V. Wang, Appl. Opt. 41, 6030 (2002); M. Gross, P. Goy, and M. Al-Koussa, Opt. Lett. 28, 2482 (2003); T. W. Murray, L. Sui, G. Maguluri, R. A. Roy, A. Nieva, F. Blonigen, and C. A. DiMarzio, Opt. Lett. 29, 2509 (2004).

[4] S. Sakadzic and L. V. Wang, Opt. Lett. 29, 2770 (2004).

[5] G. Maret and P. E. Wolf, Z. Phys. B 65, 409 (1987).

[6] W. Leutz and G. Maret, Physica (Amsterdam) 204B, 14 (1995); M. Kempe, M. Larionov, D. Zaslavsky, and A.Z. Genack, J. Opt. Soc. Am. A 14, 1151 (1997).

[7] G.D. Mahan, W.E. Engler, J.J. Tiemann, and E. G. Uzgiris, Proc. Natl. Acad. Sci. U.S.A. 95, 14015 (1998).

[8] L. V. Wang, Phys. Rev. Lett. 87, 043903 (2001).

[9] S. Sakadzic and L. V. Wang, Phys. Rev. E 66, 026603 (2002).

[10] A. Lev and B. Sfez, J. Opt. Soc. Am. A 20, 2347 (2003).

[11] S. Sakadzic and L. V. Wang, Phys. Rev. E 72, 036620 (2005).

[12] L. V. Wang, Opt. Lett. 26, 1191 (2001).

[13] D. J. Pine, D. A. Weitz, P. M. Chaikin, and E. Herbolzheimer, Phys. Rev. Lett. 60, 1134 (1988).

[14] S. Sakadzic and L. V. Wang (to be published).

[15] U. Frisch, Wave Propagation in Random Media, Probabilistic Methods in Applied Mathematics Vol. 1 (Academic, New York, 1968), pp. 75-198.

[16] Y. Barabanenkov, A. Vinogradov, Y. Kravtsov, and V. Tatarskii, Radiophys. Quantum Electron. 15, 1420 (1972); M. J. Stephen, Phys. Rev. B 37, 1 (1988).

[17] A. Ishimaru, Wave Propagation and Scattering in Random Media (Academic, New York, 1978).

[18] R. L. Dougherty, B. J. Ackerson, N. M. Reguigui, F. DorriNowkoorani, and U. Nobbmann, J. Quant. Spectrosc. Radiat. Transfer 52, 713 (1994).

[19] D. A. Boas, L. E. Campbell, and A. G. Yodh, Phys. Rev. Lett. 75, 1855 (1995).

[20] R. C. Haskell, L. O. Svaasand, T.-T. Tsay, T.-C. Feng, M. S. McAdams, and B. J. Tromberg, J. Opt. Soc. Am. A 11, 2727 (1994).

[21] M.S. Patterson, B. Chance, and B. C. Wilson, Appl. Opt. 28, 2331 (1989).

[22] J.D. Jackson, Classical Electrodynamics (Wiley, New York, 1998), 3rd ed.

[23] J. Jensen and N. B. Svendsen, IEEE Trans. Ultrason. Ferroelectr. Freq. Control 39, 262 (1992). 